This item was submitted to Loughborough's Research Repository by the author.

Items in Figshare are protected by copyright, with all rights reserved, unless otherwise indicated.

\title{
On the performance of opportunistic cooperative wireless networks
}

PLEASE CITE THE PUBLISHED VERSION

http://dx.doi.org/10.1109/TCOMM.2008.060455

PUBLISHER

(c) IEEE

VERSION

AM (Accepted Manuscript)

LICENCE

CC BY-NC-ND 4.0

REPOSITORY RECORD

Ding, Zhiguo D., Yu Gong, T. Ratnarajah, and Colin F. Cowan. 2019. "On the Performance of Opportunistic Cooperative Wireless Networks". figshare. https://hdl.handle.net/2134/25674. 


\title{
On the Performance of Opportunistic Cooperative Wireless Networks
}

\author{
Zhiguo Ding, Member, IEEE, Yu Gong, Member, IEEE, T. Ratnarajah, Senior Member, \\ IEEE and Colin Cowan, Senior Member, IEEE
}

\begin{abstract}
Relaying nodes are randomly chosen in traditional cooperative systems, which could result in severe performance loss at low SNR, specially when a large number of relaying nodes are used. Such performance loss can be avoided or alleviated by exploring a priori channel information at the transmitter. In this paper, several opportunistic relaying strategies are developed for different types of a priori channel information. By using the order statistics, explicit expression of the outage probability, as well as the diversity-multiplexing tradeoff, are developed for each proposed cooperative scheme. Our analytical results show that the more channel information available at the transmitter, the better performance a cooperative system can achieve. Especially when the source node has the full channel knowledge among the source, relays and destination, full diversity can be achieved with only one relay channel, where the optimal diversity-multiplexing tradeoff is derived as $d(r)=(N+1)(1-2 r)$, $N$ is the number of all possible relay nodes and $r$ is the transmission rate.
\end{abstract}

\section{INTRODUCTION}

A major concern in wireless communications is the signal fading at the receiver due to the multipath propagation [1]. Although multiple receive/transmit antennas can be used to combat the fading [2], [3], this well-known MIMO approach is not always feasible due to practical factors such as the size of handsets. Circumventing this problem by forming a virtue MIMO system with only a single transmit or receive antenna at each user, recent development of the cooperative networks becomes an attractive alternative [4]-[6]. The basic idea of the cooperative networks is to let other users/partners "help" to relay the information from the transmitting user to the destination, by which a mutliuser, or virtue spatial, diversity

This work was supported by the UK Engineering and Physical Sciences Research Council under grant number EP/C004132/1.

Z. Ding, T. Ratnarajah and C. Cowan are with ECIT, Queen's University of Belfast, Queen's Road, Queen's Island, Belfast, BT3 9DT, UK; Email: Z.Ding@ecit.qub.ac.uk, T.Ratnarajah@ieee.org, C.F.N.Cowan@ee.qub.ac.uk; Phone: +44 289097 1890; Fax: +44 289097 1702. Y. Gong is with the School of Systems Engineering, University of Reading, RG6 6AY, Reading, UK. emai l : y.gong@reading.co.uk 
is achieved. Such cooperative approach is based on a fact that the antennas used in a wireless system are omnidirectional, making it possible for other users to overheard and relay the signals transmitted towards the destination.

The protocol, or the relay scheme, is the core to a cooperative networks. Depending on whether the relay user decodes the information or not, cooperative protocols may be classified as decode-and-forward or amplified-and-forward type. Cooperative protocols described in [4] are among the first that have been systematically studied. These relay schemes are known to have better performance than direct transmission at high SNR, but minor performance gain at low SNR because of the extra channel resource consumed by the relay users. Such unbalanced performance phenomenon becomes more serious with the increase of the relay nodes participating in the transmission. As was shown in [7], for example, a cooperative scenario with a large number of relaying nodes suffers from severe performance loss at low SNR, being even worse than direct transmission. To make the problem even worse, some of the relay nodes may not be able to forward the source information reliably. Therefore, the diversity achieved by cooperation comes with the price of the loss of bandwidth efficiency.

Hence one challenging task in cooperative networks design is to minimize the bandwidth resource consumed by the relay users. One spectrally efficient way is to use non-orthogonal transmission and allow several users transmit simultaneously [8], [9], which, however, can result some difficulties in the design of medium access control protocols. Another intuitive approach is to let the relay occur only when the direct transmission becomes unreliable, and, when the relay is necessary, to ask only a necessary number of the relays to participate in the communications. With the control of the relays, the spectral efficiency can be improved, depending on how much a priori information can be used by the source node. This problem was first studied in [10], [11] and will be investigated in detail in this paper.

To be specific, we will consider five cooperative schemes, ranging from the case that no a priori channel information is known at the source node, to the ideal case that full channel knowledge are available. Except for the first case that no a-priori information is available since it has been studied in [7], in this paper, the explicit expressions of the outage probability at arbitrary SNR, as well as the the diversity-multiplexing tradeoff [12], [13] at at high SNR, for all other scenarios are developed, which are later shown fit well with the Monte-Carlo simulations. The developed analytical results prove the intuition that the more the a priori channel information available, the higher spectral efficiency the cooperative system can achieve. Moreover, we also show that, except for the ideal case that the full diversity can be achieved with only only one relay link, for all other cases, the more the relay links that joins in the transmission, the larger the diversity that the system can reach. These results imply that, though it is a good performance index at the high SNR, the multiplexing-diversity tradeoff is not an approximate one at the low SNR. Hence 
the same high SNR behavior does not always promise the same performance at the low SNR.

The rest of the paper is organized as follows: Five cooperative protocols are described in Section II; The outage probability for the five protocols are derived from Section III to VI respectively. Numerical results are given in Section VII. Mathematical proofs are collected in the Appendix. Finally, Section VIII concludes the paper.

\section{Cooperative Protocals}

To simplify the clarification, the protocols studied in this paper are based on time division schemes as did in [4], where each user is assigned a unique time slot which is further divided into several sub-time slots. As an illustration, Fig. 1 shows the details of the first time slot, where user 1 is the transmitting user and the others are the relay users. During the sub-time slot 1 , only the user 1 transmits, while the other users keep silence by "listening". During the following sub-time slots, the relays then forward the overheard signals to the destination. In this paper, we mainly consider the decode-and-forward type of the schemes, i.e. the relay nodes decode the information before they are forwarded, if necessary, to the destination.

\section{Time slot 1}

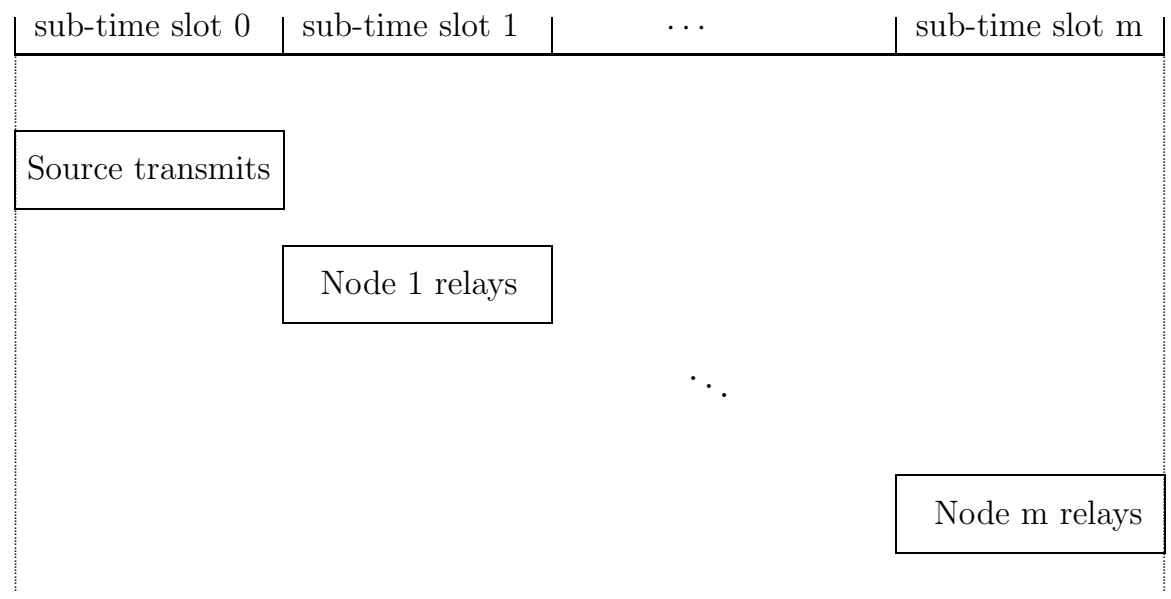

Fig. 1. The slot assignment for the time slot I.

To improve the spectral efficiency, a priori channel information is explored by the source node. According to how much system overhead caused by feedback is allowed, the a priori channel state information (CSI) acquired at the source node can be categorized into five cases as shown in Table I. The reason why the transmitter can have two types of information for one channel measurement can be clarified as the following. Due to the time-varying nature of wireless fading channels, the instantaneous channel measurements feedbacked from the receivers might not be valid, whereas the order of the channels 
can still be accurate. Here we assume that the transmitter can have the access to the channel information as discussed in [11].

TABLE I

TYPE OF PRIORI CHANNEL INFORMATION

\begin{tabular}{c|c|c|c|c}
\hline Type & $\begin{array}{c}\text { Order information } \\
\text { Channel } \mathrm{S} \rightarrow \mathrm{R}\end{array}$ & $\begin{array}{c}\text { Exact value } \\
\text { Channel } \mathrm{S} \rightarrow \mathrm{R}\end{array}$ & $\begin{array}{c}\text { Exact value } \\
\text { Channel } \mathrm{S} \rightarrow \mathrm{D}\end{array}$ & $\begin{array}{c}\text { Exact value } \\
\text { Channel } \mathrm{R} \rightarrow \mathrm{D}\end{array}$ \\
\hline I & No & No & No & No \\
\hline II & Yes & No & No & No \\
\hline III & Yes & Yes & No & No \\
\hline IV & Yes & Yes & Yes & No \\
\hline V & Yes & Yes & Yes & Yes \\
\hline
\end{tabular}

To fully utilize the available information, different cooperative strategies should be developed as described below.

- Random n-Relay Scheme with Type I CSI: With no a priori CSI available at all, a random $n$ number of relays are chosen.

- Opportunistic n-Relay Scheme with Type II CSI: With the order information of the source-relay channels, the best $n$ number of the relays are chosen.

- Opportunistic Relay Scheme with Type III CSI: With the exact CSI values from the source to relay nodes, only the relay nodes which correctly decode the source information participate in the communications.

- Opportunistic Incremental Scheme with Type IV CSI: Beside the source to relay, the exact CSI value from source to destination is also available. The source first decides whether it needs cooperative communication or not. If it does, the scheme operates in the same manner as that for the Type III CSI.

- Opportunistic Best-Relay Scheme with Type V CSI: With full knowledge of all channels among the source, relays and destination, only the relay nodes having good links with both the source and destination are chosen.

The Random $n$-Relay scheme has been studied in [7]. This paper will focus on the other four schemes. We will show that, when only the order information of the source-relaying channels is available, the cooperative system still suffers from performance loss at low SNR. Only when the exact value of the source-relaying channels becomes available, can the performance loss at the low SNR be effectively reduced, where the full diversity is reached with all of the possible relay nodes being participating in the communications. On the other hand, for the ideal case that the source node have the full information 
of all channels, full diversity is achieved with only one relays. These observations match well with our original intuition that the more the a priori channel information that available at the source, the better performance we can achieve.

\section{OpPORTUNistiC $n$-RELAY SCHEME WITH TYPE II CSI}

Consider that the source node has the order information of the channels between the source and relay nodes. It will be a natural choice to only use the $n$ relay nodes with the best channel conditions. Hence an interesting question is what is the effect of the choice $n$ to the whole system performance. Define $x_{i}=\left|h_{s i}\right|^{2}$ as the channel from the source to the $i$ th relay nodes and assume

$$
x_{(1)} \leq x_{(2)} \leq \cdots \leq x_{(N-1)} \leq x_{(N)}
$$

Provided that only the $n$ best relay nodes are chosen to participate in communications. Since the exact values of CSI are unknown, the channels between the source and the chosen $n$ relays are still possible suffer deep fading. Assume the chosen relaying node can forward the source information if $x_{i}>\frac{2^{(n+1) R}-1}{\rho},(N-n+1) \leq i \leq N$, otherwise it keeps silent. The following theorem gives the outage probability of this opportunistic scheme.

Theorem 1: The outage probability of the opportunistic $n$-relay scheme with type II CSI can be expressed as

$$
\begin{aligned}
P_{\text {out }, I}= & \sum_{k=0}^{n-1}\left[1-e^{-\lambda \gamma_{n}} \sum_{i=0}^{k} \frac{\left(\lambda \gamma_{n}\right)^{i}}{i !}\right] \frac{N !}{(N-k) !(k) !}\left[1-e^{-\lambda \gamma_{n}}\right]^{N-k}\left[e^{-\lambda \gamma_{n}}\right]^{k}+ \\
& {\left[1-e^{-\lambda \gamma_{n}} \sum_{i=0}^{n} \frac{\left(\lambda \gamma_{n}\right)^{i}}{i !}\right] \sum_{m=n}^{N} \frac{N !}{(N-m) !(m) !}\left[1-e^{-\lambda \gamma_{n}}\right]^{N-m}\left[e^{-\lambda \gamma_{n}}\right]^{m} }
\end{aligned}
$$

and the outage probability can be approximated at high SNR as

$$
P_{\text {out }, I} \approx \frac{\left[\lambda \gamma_{n}\right]^{n+1}}{(n+1) !} \sum_{m=n}^{N} \frac{N !}{(N-m) !(m) !}
$$

where $\gamma_{n}=\frac{2^{(n+1) R}-1}{\rho}$

Proof: See Appendix

With the help of the developed outage probability, the diversity-multiplexing tradeoff can be obtained as the following.

Theorem 2: The diversity-multiplexing tradeoff of the opportunistic $n$-relay scheme with type II CSI can be expressed as

$$
d(r)=[n+1][1-(n+1) r]
$$


Proof: The diversity-multiplexing tradeoff of the system can be obtained as

$$
\begin{aligned}
d(r) & =\quad-\lim _{\rho \rightarrow \infty} \frac{\log P_{\text {out }}}{\log \rho} \\
& =\quad-\lim _{\rho \rightarrow \infty} \frac{\log \left[\lambda \frac{2^{(n+1) R-1}}{\rho}\right]^{n+1} C}{\log \rho} \\
& =\quad-\lim _{\rho \rightarrow \infty} \frac{\log \left[\lambda \frac{\rho^{(n+1) r-1}}{\rho}\right]^{n+1}+\log C}{\log \rho} \\
& \approx \quad-\lim _{\rho \rightarrow \infty} \frac{\left[\rho^{(n+1) r-1}\right]^{n+1}}{\log \rho} \\
& =\quad[n+1][1-(n+1) r],
\end{aligned}
$$

where $C=\sum_{m=n}^{N} \frac{N !}{(N-m) !(m) !}$.

Theorem 1 and 2 reveal that a cooperative system should use all possible relaying nodes to achieve the full diversity $N+1$ when only the order information of the source-to-relaying channels is available. But the increase of the number of the used relay nodes will result in the the loss of the multiplexing gain as shown in the simulation section.

\section{OpPoRTUNistic Relay SCHEME With TyPE III CSI}

With Type III CSI, the source node has the access to not only the order information, but also the exact values of the channel between the source and the relay nodes. Provided that there will be $n$ relay nodes satisfying the desired threshold, the question of interest is whether all or part of the $n$ nodes should be chosen, which will be discussed in the following two subsections.

\section{A. Opportunistic n-Relay Scheme with Type III CSI}

Assume that there are $n$ relay nodes which can decode the source information successfully, and all these $n$ nodes are used for cooperation. The following theorem gives the outage probability of this cooperative scheme.

Theorem 3: Consider that Type III CSI is available at the source node and all $n$ qualified relaying nodes will participate in communications. The outage probability of such opportunistic relay scheme can be expressed as

$$
\begin{aligned}
P_{\text {out }}= & \sum_{n=1}^{N-1}\left[1-e^{-\lambda \gamma_{n}} \sum_{i=0}^{n} \frac{\left[\lambda \gamma_{n}\right]^{i}}{i !}\right] \frac{N !}{(N-n) ! n !}\left[1-e^{-\lambda \gamma_{n+1}}\right]^{N-n}\left[e^{-\lambda \gamma_{n}}\right]^{n} \\
& +\left[1-e^{-\lambda \gamma_{0}}\right]\left[1-e^{-\lambda \gamma_{1}}\right]^{N}+\left[1-e^{-\lambda \gamma_{N}} \sum_{i=0}^{N} \frac{\left[\lambda \gamma_{N}\right]^{i}}{i !}\right]\left[e^{-\lambda \gamma_{N}}\right]^{N}
\end{aligned}
$$


and the outage probability can be approximated at high SNR as

Proof: See Appendix

$$
P_{\text {out }} \approx \sum_{n=1}^{N-1} \frac{N !}{(N-n) ! n !} \frac{\left[\lambda \gamma_{n}\right]^{n+1}}{(n+1) !}\left[\lambda \gamma_{n+1}\right]^{N-n}+\lambda \gamma_{0}\left[\lambda \gamma_{1}\right]^{N}+\frac{\left[\lambda \gamma_{N}\right]^{N+1}}{(N+1) !}
$$

Theorem 4: Consider that Type III CSI is available at the source node and all $n$ qualified relaying nodes will participate in communications. The diversity-multiplexing tradeoff of the opportunistic relay scheme can be expressed as

$$
d(r)=[N+1][1-(N+1) r]
$$

Proof: By substituting $R=r \log \rho$ into the expression of the outage probability, we obtain

$$
\begin{aligned}
P_{\text {out }}(r) \approx & \sum_{n=1}^{N-1} \frac{N !}{(N-n) ! n !} \frac{\left[\lambda\left(\rho^{(n+1) r}-1\right)\right]^{n+1}}{(n+1) ! \rho^{N+1}}\left[\lambda\left(\rho^{(n+2) r}-1\right)\right]^{N-n} \\
& +\lambda \frac{\left(\rho^{r}-1\right)}{\rho^{N+1}}\left[\lambda\left(\rho^{2 r}-1\right)\right]^{N}+\frac{\left[\lambda\left(\rho^{(N+1) r}-1\right)\right]^{N+1}}{(N+1) ! \rho^{N+1}} .
\end{aligned}
$$

For high SNR, $\rho \rightarrow \infty$, the last factor of (9) will dominate the equation and hence the tradeoff can be obtained by the following the steps in Proof of Theorem 2.

\section{B. Opportunistic m-Relay Scheme with Type III CSI}

Provided the existence of the $n$ qualified relay nodes, only $m$ of these nodes are chosen to participate communication. Note that $m$ is a pre-fixed choice, but $n$ will be varying according to the channel condition. In case that $n$ is smaller than $m$, all of the $n$ nodes will be chosen. The following theorem gives the outage probability of this relaying scheme.

Theorem 5: Consider that Type III CSI is available at the source node and only $m$ of the $n$ qualified relaying nodes will participate in communications. The outage probability of the opportunistic relay scheme can be expressed as

$$
\begin{aligned}
P_{\text {out }}= & {\left[1-e^{-\lambda \gamma_{m}} \sum_{i=0}^{m} \frac{\left[\lambda \gamma_{m}\right]^{i}}{i !}\right]\left[\sum_{k=m}^{N-1} \frac{N !}{(N-k) ! k !}\left[1-e^{-\lambda \gamma_{k+1}}\right]^{N-k}\left[e^{-\lambda \gamma_{k}}\right]^{k}+\left[e^{-\lambda \gamma_{N}}\right]^{N}\right] } \\
& +\sum_{n=1}^{m-1}\left[1-e^{-\lambda \gamma_{n}} \sum_{i=0}^{n} \frac{\left[\lambda \gamma_{n}\right]^{i}}{i !}\right] \frac{N !}{(N-n) ! n !}\left[1-e^{-\lambda \gamma_{n+1}}\right]^{N-n}\left[e^{-\lambda \gamma_{n}}\right]^{n} \\
& +\left[1-e^{-\lambda \gamma_{0}}\right]\left[1-e^{-\lambda \gamma_{1}}\right]^{N}
\end{aligned}
$$

and the outage probability can be approximated at high SNR as

$$
P_{\text {out }} \approx \frac{\left[\lambda \gamma_{m}\right]^{m+1}}{(m+1) !} \sum_{k=m}^{N-1} \frac{N !}{(N-k) ! k !}\left[\lambda \gamma_{k+1}\right]^{N-k}+\sum_{n=1}^{m-1} \frac{N !}{(N-n) ! n !} \frac{\left[\lambda \gamma_{n}\right]^{n+1}}{(n+1) !}\left[\lambda \gamma_{n+1}\right]^{N-n}+\left[\lambda \gamma_{0}\right]\left[\lambda \gamma_{1}\right]^{N}
$$


Proof: Since the $m$ nodes are chosen from the $n$ qualified nodes, the outage event will be either caused by the poor link quality between the relay and destination nodes, or there is no qualified relay node existing $n=0$. Hence the outage probability can be written as

$$
\begin{aligned}
P_{\text {out }} & =P\left(\mathcal{I}_{m}<\gamma_{m} \mid n \geq m\right) P(n \geq m)+\sum_{n=0}^{m-1} P\left(\mathcal{I}_{n}<\gamma_{n} \mid \mathbf{n}=n\right) P(\mathbf{n}=n) \\
& =P\left(\mathcal{I}_{m}<\gamma_{m} \mid n \geq m\right) \sum_{k=m}^{N} P(\mathbf{n}=k)+\sum_{n=0}^{m-1} P\left(\mathcal{I}_{n}<\gamma_{n} \mid \mathbf{n}=n\right) P(\mathbf{n}=n)
\end{aligned}
$$

Note that the probability of the event $\mathbf{n}=k$ and $\mathcal{I}_{m}<\gamma_{m}$ can be obtained from (47) and (48), and hence the theorem can be easily proved.

After obtaining the outage probability, the diversity-multiplexing tradeoff will be found by following the similar steps in the proof for Theorem 2 and given by the following theorem.

Theorem 6: Consider that Type III CSI is available at the source node and the $m$ of the $n$ qualified relaying nodes will participate in communications. The diversity-multiplexing tradeoff of the opportunistic relay scheme can be expressed as

$$
d(r)=[m+1][1-(m+1) r] .
$$

From Theorem 4 and Theorem 6, we have the following two remarks.

(i) Theorem 4 shows that the $n$-relay scheme can promise the full diversity $N+1$ at high SNR. The reason is that at high enough SNR, all of the $N$ possible relay nodes can receive the source information correctly and hence the choice of $n$ will be $N$ naturally.

(ii) The multiplexing-diversity tradeoff of the $m$-relay scheme with Type III CSI is the same as the one with Type II CSI, which means that the two scheme have the same high SNR behavior. But such same behavior at high SNR does not promise their same performance at low SNR. Note that now more channel information is available at the transmitter. So the chosen relaying nodes can be assured effective and hence the outage probability at low SNR can be well suppressed as shown in the simulation section.

\section{OPPORTUNistic Relay InCREMENTAL SCHEME With TyPe IV CSI}

Compared with the previous schemes, Type IV CSI provides additional information that the source node knows the channel between the source and destination nodes. As did in [4], the source node will first decide whether it needs help from other nodes by comparing its link with the destination to the desired threshold. Then with help of the knowledge of CSI, the source node will select which and how many nodes to participate in communications. Similar to the previous scheme, there are two options of the node selection, whose outage probability is given by the following two subsections. 


\section{A. Opportunistic n-Relay Incremental Scheme with Type IV CSI}

Assume that there are $n$ relay nodes which can decode the source information successfully, and all these $n$ nodes are used for cooperation. The following theorem gives the outage probability of this cooperative scheme.

Theorem 7: Consider that Type IV CSI is available at the source node and all of the $n$ qualified relaying nodes will participate in communications. The outage probability of the opportunistic relay scheme can be expressed as

$$
\begin{aligned}
P_{\text {out }}= & \sum_{n=1}^{N-1}\left\{\left[1-e^{-\lambda \gamma_{0}}\right] \Psi\left(n-1, \gamma_{n}-\gamma_{0}\right)+\Psi\left(n, \gamma_{n}\right) e^{-\lambda\left[\gamma_{n}-\gamma_{0}\right]} \sum_{i=0}^{n-1} \frac{\left[\lambda\left(\gamma_{n}-\gamma_{0}\right)\right]^{i}}{i !}\right\} \\
& \times \frac{N !}{(N-n) ! n !}\left[1-e^{-\lambda \gamma_{n+1}}\right]^{N-n}\left[e^{-\lambda \gamma_{n}}\right]^{n} \\
& +\left\{\left[1-e^{-\lambda \gamma_{0}}\right] \Psi\left(N-1, \gamma_{N}-\gamma_{0}\right)+\Psi\left(N, \gamma_{n}\right) e^{-\lambda\left[\gamma_{N}-\gamma_{0}\right]} \sum_{i=0}^{N-1} \frac{\left[\lambda\left(\gamma_{N}-\gamma_{0}\right)\right]^{i}}{i !}\right\}\left[e^{-\lambda \gamma_{N}}\right]^{N} \\
& +\left[1-e^{-\lambda \gamma_{0}}\right]\left[1-e^{-\lambda \gamma_{1}}\right]^{N}
\end{aligned}
$$

where $\Psi(n, z)=\left[1-e^{-\lambda z} \sum_{i=0}^{n} \frac{[\lambda z]^{i}}{i !}\right]$ and the outage probability can be approximated at high SNR as

$$
P_{\text {out }} \approx \sum_{n=1}^{N} \frac{N !}{(N-n) ! n !}\left[\lambda \gamma_{n+1}\right]^{N-n}\left[\frac{\left[\lambda\left(\gamma_{n}-\gamma_{0}\right)\right]^{n}}{n !} \lambda \gamma_{0}+\frac{\left[\lambda \gamma_{n}\right]^{n+1}}{(n+1) !}\right]+\lambda \gamma_{0}\left[\lambda \gamma_{1}\right]^{N}
$$

Proof: For the addressed scheme, all relay nodes which can forward the source information correctly will be chosen to participate into communications. Hence the outage probability can be written as

$$
\begin{aligned}
P_{\text {out }} & =\sum_{n=0}^{N} P\left(\mathcal{I}_{n}<R \mid \mathbf{n}=n, \mathcal{I}_{D}<R\right) P\left(\mathbf{n}=n, \mathcal{I}_{D}<R\right) \\
& =\sum_{n=0}^{N} P\left(\mathcal{I}_{n}<R, \mathcal{I}_{D}<R \mid \mathbf{n}=n\right) P(\mathbf{n}=n)
\end{aligned}
$$

where the threshold is now a function of the number of the used relay node.

The first factor of the product in (16) can be written as

$$
\begin{aligned}
P\left(\mathcal{I}_{n}<R, \mathcal{I}_{D}<R \mid \mathbf{n}=n\right)= & P\left(x_{0}+x_{\text {sum }}<\gamma(n), x_{0}<\gamma(0) \mid \mathbf{n}=n\right) \\
= & P\left[x_{0}<\gamma(0)\right] P\left[\gamma(n)-x_{\text {sum }}>\gamma(0) \mid \mathbf{n}=n\right] \\
& +P\left[x_{0}<\gamma(0)-x_{\text {sum }} \mid \mathbf{n}=n\right] P\left[\gamma(n)-x_{\text {sum }}<\gamma(0) \mid \mathbf{n}=n\right] \\
= & P\left[x_{0}<\gamma(0)\right] P\left[x_{\text {sum }}<\gamma(n)-\gamma(0) \mid \mathbf{n}=n\right] \\
& +P\left[x_{0}+x_{\text {sum }}<\gamma(n) \mid \mathbf{n}=n\right] P\left[x_{\text {sum }}>\gamma(n)-\gamma(0) \mid \mathbf{n}=n\right]
\end{aligned}
$$

where $x_{\text {sum }}=\sum_{i=1}^{n} x_{i}$. The evaluation of the probability requires the PDF of $x_{0}, x_{\text {sum }}$ and $x_{0}+x_{\text {sum }}$ which have been obtained previously. Hence the theorem is proved. 
Similar to the previous relay scheme, the diversity-multiplexing tradeoff can be written as

Theorem 8: Consider that Type IV CSI is available at the source node and all of the $n$ qualified relaying nodes will participate in communications. The diversity-multiplexing tradeoff of the opportunistic relay scheme can be expressed as

$$
d(r)=[N+1][1-(N+1) r] .
$$

\section{B. Opportunistic m-Relay Scheme with Type IV CSI}

Provided the existence of the $n$ qualified relay nodes, only $m$ of these nodes are chosen to participate communication. The following theorem gives the outage probability of this relaying scheme.

Theorem 9: Consider that Type IV CSI is available at the source node and only $m$ of the $n$ qualified relaying nodes will participate in communications. The outage probability of the opportunistic relay scheme CSI can be expressed as

$$
\begin{aligned}
P_{\text {out }}= & \left\{\left[1-e^{-\lambda \gamma_{0}}\right] \Psi\left(m-1, \gamma_{m}-\gamma_{0}\right)+\Psi\left(m, \gamma_{m}\right) e^{-\lambda\left[\gamma_{m}-\gamma_{0}\right]} \sum_{i=0}^{m-1} \frac{\left[\lambda\left(\gamma_{m}-\gamma_{0}\right)\right]^{i}}{i !}\right\} \\
& \times\left[\sum_{k=m}^{N-1} \frac{N !}{(N-k) ! k !}\left[1-e^{-\lambda \gamma_{k+1}}\right]^{N-k}\left[e^{-\lambda \gamma_{k}}\right]^{k}+\left[e^{-\lambda \gamma_{N}}\right]^{N}\right] \\
& +\sum_{n=1}^{m-1}\left\{\left[1-e^{-\lambda \gamma_{0}}\right] \Psi\left(n-1, \gamma_{n}-\gamma_{0}\right)+\Psi\left(n, \gamma_{n}\right) e^{-\lambda\left[\gamma_{n}-\gamma_{0}\right]} \sum_{i=0}^{n-1} \frac{\left[\lambda\left(\gamma_{n}-\gamma_{0}\right)\right]^{i}}{i !}\right\} \\
& \times \frac{N !}{(N-n) ! n !}\left[1-e^{-\lambda \gamma_{n+1}}\right]^{N-n}\left[e^{-\lambda \gamma_{n}}\right]^{n}+\left[1-e^{-\lambda \gamma_{0}}\right]\left[1-e^{-\lambda \gamma_{1}}\right]^{N}
\end{aligned}
$$

where $\Psi(n, z)=\left[1-e^{-\lambda z} \sum_{i=0}^{n} \frac{[\lambda z]^{i}}{i !}\right]$ and the outage probability can be approximated at high SNR as

$$
\begin{aligned}
P_{\text {out }} \approx & {\left[\lambda \gamma_{0} \frac{\left[\lambda\left(\gamma_{m}-\gamma_{0}\right)\right]^{m}}{m !}+\frac{\left[\lambda \gamma_{m}\right]^{m+1}}{(m+1) !}\right] \sum_{k=m}^{N-1} \frac{N !}{(N-k) ! k !}\left[\lambda \gamma_{k+1}\right]^{N-k} } \\
& +\sum_{n=1}^{m-1} \frac{N !}{(N-n) ! n !}\left[\lambda \gamma_{n+1}\right]^{N-n}\left[\lambda \gamma_{0} \frac{\left[\lambda\left(\gamma_{n}-\gamma_{0}\right)\right]^{n}}{n !}+\frac{\left[\lambda \gamma_{n}^{n+1}\right]}{(n+1) !}\right]+\lambda \gamma_{0}\left[\lambda \gamma_{1}\right]^{N}
\end{aligned}
$$

Proof: The theorem can be proved by coming the proof for Theorem 5 and 9.

Similar to the previous relay scheme, the diversity-multiplexing tradeoff can be written as

Theorem 10: Consider that Type IV CSI is available at the source node and only $m$ of the $n$ qualified relaying nodes will participate in communications. The diversity-multiplexing tradeoff of the opportunistic relay scheme can be expressed as

$$
d(r)=[m+1][1-(m+1) r]
$$




\section{Opportunistic Best-Relay Scheme with Full CSI}

With the help of Type V CSI, the source node has the priori information of the source-to-relay channels as well as the relay-to-destination channels. Hence the strategy of the best-relaying scheme can be described as the following. First the source node will select the $n$ relay nodes which have the qualified source-to-relay link quality. Define $z_{i}=\left|\bar{h}_{i}\right|^{2}, 1 \leq i \leq n$, are the fading coefficients corresponding to the channel from the $n$ selected relay nodes to the destination. Assume these corresponding relay-to-destination channels are ordered as

$$
z_{(1)} \leq z_{(2)} \leq \cdots \leq z_{(n-1)} \leq z_{(n)}
$$

The source node will choose the node with the best relay-to-destination link quality. Hence the propose scheme will only use one relay node which have the best relay-to-destination link quality as well as the qualified source-to-relay link. The following theorem gives the outage probability of this scheme.

Theorem 11: Consider that Type V CSI is available at the source node. The outage probability of the opportunistic best-relay scheme can be expressed as

$$
\begin{aligned}
P_{\text {out }}= & \sum_{n=0}^{N} \frac{N ! \lambda}{(N-n) ! n !}\left[1-e^{-\lambda \gamma(1)}\right]^{N-n}\left[e^{-\lambda \gamma(1)}\right]^{n+1} \\
& \times\left\{\sum_{\substack{i=0 \\
i \neq 1}}^{n} C_{n}^{i}(-1)^{i} \frac{1}{(i-1) \lambda}\left[1-e^{-(i-1) \lambda \gamma_{1}}\right]-n \lambda \gamma_{1}\right\}
\end{aligned}
$$

and the outage probability can be approximated at high SNR as

Proof: See Appendix.

$$
P_{\text {out }} \approx[\lambda \gamma(1)]^{N+1} \sum_{n=1}^{N-1} \frac{N !}{(N-n) !(n+1) !}
$$

With the help of the developed outage probability, the diversity-multiplexing tradeoff can be easily obtained as given by the following theorem.

Theorem 12: Consider that Type V CSI is available at the source node. The diversity-multiplexing tradeoff of the opportunistic best-relay scheme can be expressed as

$$
d(r)=[N+1][1-2 r] .
$$

Theorem 11 and 12 provide an interesting result that the best-relay scheme can achieve the full diversity by only using one extra channel use for relaying transmission, which can be illustrated by the following clarification. Consider all of $N$ possible nodes are used which of course can have the best reliability of reception. Provided that there $n$ relaying nodes can decode the source information correctly. A outage event that all of the $n$ relay-destination channel links are falling down the desired threshold equals to the event that the best one among the $n$ relay-destination links is not qualified. So the best-relay scheme can 
achieve the same reliability of reception as the scheme which uses all $N$ nodes. But the best-relay scheme is more spectrally efficient as it only uses one extra channel use.

\section{NUMERICAL RESULTS}

In this section, simulation results are presented to demonstrate the performance of the proposed relaying scenarios as well as the accuracy of the developed analytical results. The number of the possible relaying nodes is $N=5$. The data rate is set as $R=1 \mathrm{bit} / \mathrm{sec} / \mathrm{Hz}$.

In the first experiment, we compare the performance of the proposed strategy with Type II CSI with the scheme in [7] which is the multiple-node extension of the "classical" scheme in [4] and dose not utilize any channel information at the transmitter. As can be seen from Fig. 2, the proposed scheme utilizing the priori channel information can achieve better performance than the compared protocol. Another interesting fact observing from Fig. 2 is that the less nodes chosen, the bigger is the performance gap between the two strategies. If only one node is used, the proposed scheme can achieve around $5 \mathrm{~dB}$ performance gain over the compared protocol. And the both strategies will have the same performance if all of 5 nodes are chosen, which is due to the fact there is no difference between the cases with and without the ordered channel information.

In Fig 3, we show the outage probability of the proposed relaying scheme with Type II CSI as a function of SNR. The accuracy of the developed analytical expressions is demonstrated by the fact that the curve of Monte Carlo simulation is very close to the analytical ones. As can be seen from the figure, at the high SNR region, the proposed method can achieve better performance than the direct transmission scheme, and the number of the used relaying node is proportional to the superiority of the system performance. However, at the low SNR region, an interesting phenomenon is observed that more nodes participating in communication results in larger outage probability. Note that similar results were also reported in [7]. Such contradicted performance can be clarified that the more relay partners chosen, the more channel uses will be consumed by relaying transmission. Recall that these chosen relaying nodes may be unable to retransmit the source information and only waste the allocated bandwidth source. This is the reason for the performance penalty at low SNR although incorporating more nodes can achieve larger diversity order. Note that such unbalanced performance behavior will make a practical cooperative system difficult to design. The number of the used relaying nodes has to be chosen carefully and there will be a tradeoff between the low SNR performance loss and high SNR performance gain.

As pointed out in Section IV, the knowledge of the exact values of CSI can suppress the loss of the system performance at low SNR. In Fig 4 the performance of the proposed relaying scheme with Type III CSI is demonstrated as a function of SNR. Similar to the previous figure, the curves of analytical results are very close to the simulation results, which demonstrates the accuracy of the analytical expressions. Also 
as expected, the performance of the cooperative system will be generally increased with the number of the used relaying nodes, both at high and low SNR. In Fig 5 the performance of the proposed incremental scheme with Type IV CSI is also shown as a function of SNR, where similar relationship between the outage probability and the number of the used relay is also observed. Since the additional information of the source-to-destination channel is known, there will be slight performance gain between the scheme with Type III CSI and the one with Type IV CSI.

In Fig. 6 the performance of the best-relay scheme is shown as well as the comparison of the proposed relaying protocols and direct transmission. Since the source node has the access to the all channel information of both the source-to-relay and relay-to-destination, it is reasonable that the best-relay scheme can achieve the best performance. It is interesting to observe that the curves of the best-relay scheme, the five-relay scheme with Type II CSI and the five-relay scheme with Type III CSI have the same slop, which is due to the fact that all of them can achieve the full diversity order $d=5$. Both the best-relay scheme and the protocol with Type III/IV CSI can achieve stable performance at all SNR, but the relaying scheme with Type II CSI can not work at the SNR region $0 \leq \rho \leq 15$ which could be a working region in a practical wireless system.

\section{CONCLUSION}

In this paper we have studied a cooperative communication system where some priori information of wireless channels is available at the transmitter. Traditionally relaying nodes are randomly chosen, which could result in severe performance loss at low SNR, specially when a large number of relaying nodes are used. To fully incorporate the available channel information, several opportunistic relaying strategies are developed. Then an explicit expression of the outage probability is developed for each proposed cooperative schemes, which was shown close to the provided Monte Carlo simulation. The high SNR approximation of the outage probability as well as the diversity-multiplexing tradeoff were also developed. Our analytical results showed that the more channel information available at the transmitter, the better performance a cooperative system can achieve. When the exact values of the source-relay channels are available, the performance loss at low SNR can be effectively suppressed. And the full diversity $N+1$ can be achieved by only costing one extra channel use where the source node has the access to the source-relay and relay-destination channels.

\section{APPENDIX}

Proof for Theorem 1: Recall that only the $n$ best relay nodes are chosen to participate in communications. Since the exact values of CSI are unknown, the channels between the source and the chosen $n$ relay are still possible suffer deep fading. It is assumed that the chosen relaying node can forward the 


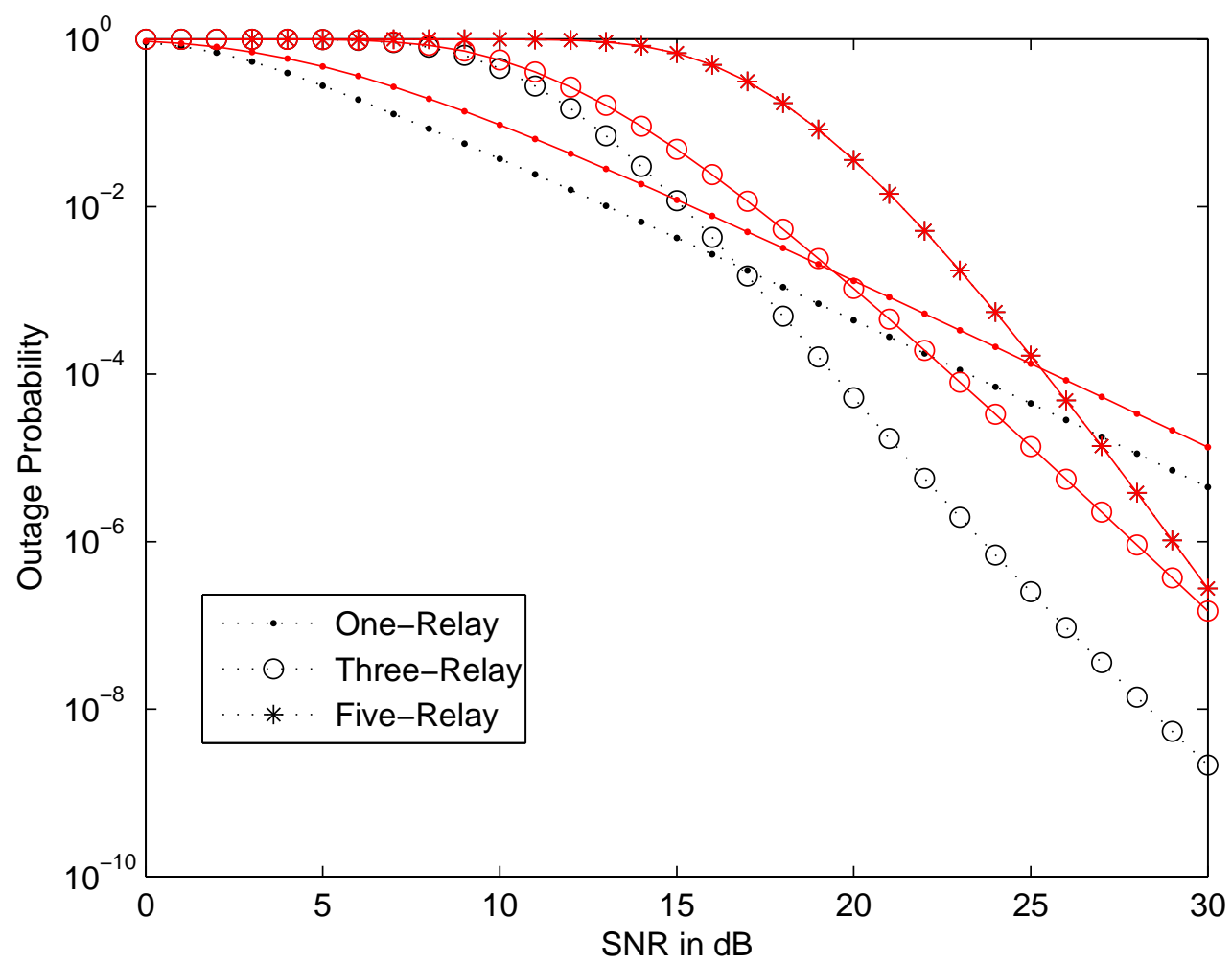

Fig. 2. The outage probability vs SNR. The dotted lines are for the scheme with no priori information of CSI and the solid lines are for the proposed relaying scheme with Type II CSI.

source information if $x_{(i)}>\gamma_{n}$, otherwise it keeps silent. Hence there will be $n+1$ possible values for the number of the successful relay nodes.

$$
\begin{array}{rcll}
\mathcal{O}_{0}: & x_{(N)}<\gamma_{n} & & \\
\mathcal{O}_{1}: & x_{(N-1)}<\gamma_{n} & \& & x_{(N)}>\gamma_{n} \\
\vdots & \vdots & \vdots & \\
\mathcal{O}_{k}: & x_{(N-k)}<\gamma_{n} & \& & x_{(N-k+1)}>\gamma_{n} \\
\vdots & \vdots & \vdots & \\
\mathcal{O}_{n-1}: & x_{(N-n+1)}<\gamma_{n} & \& & x_{(N-n+2)}>\gamma_{n} \\
\mathcal{O}_{n}: & & & x_{(N-n+1)}>\gamma_{n}
\end{array}
$$

The outate probability of the system can be expressed as

$$
P_{\text {out }, I}=\sum_{k=0}^{n} P\left(I<R \mid \mathcal{O}_{k}\right) P\left(\mathcal{O}_{k}\right) .
$$

Provided $\mathcal{O}_{k}$ happened, the data model can be written as

$$
\mathbf{y}=\mathbf{h} s+w,
$$




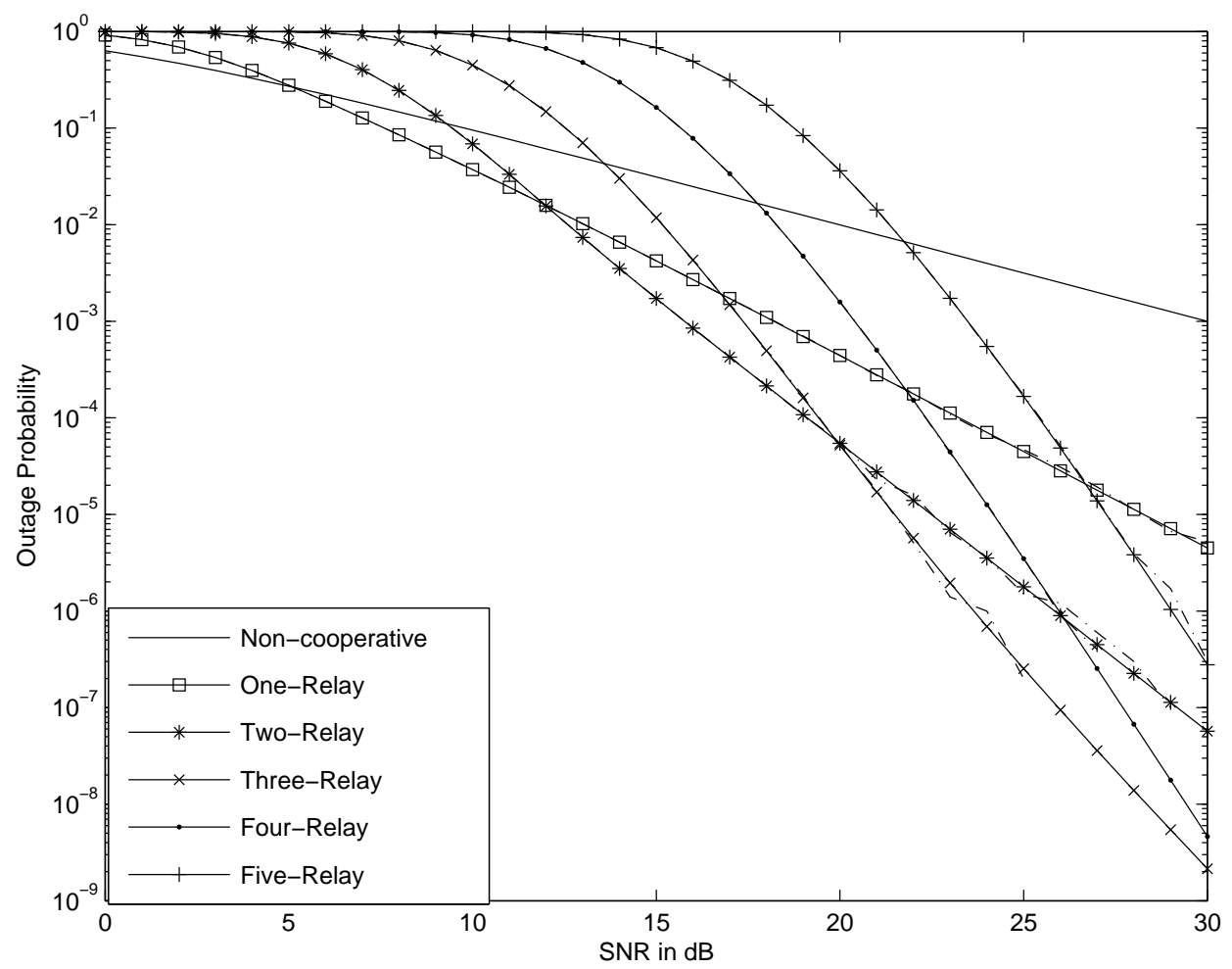

Fig. 3. The outage probability of the relaying scheme with Type II CSI vs SNR. The dashed lines are for the Monte Carlo simulation and the solid lines are for the analytical results.

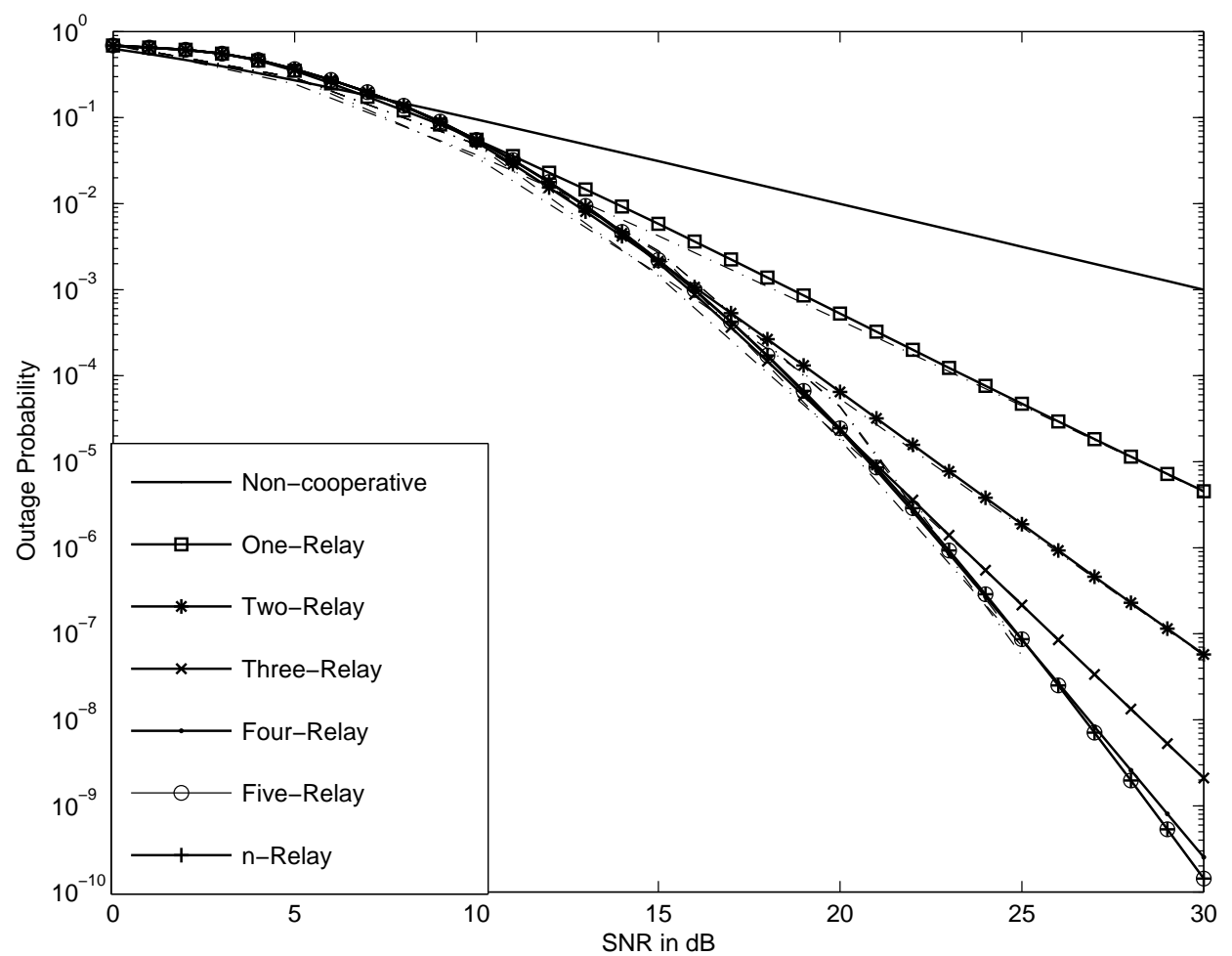

Fig. 4. The outage probability of the relaying scheme with Type III CSI vs SNR. The dotted lines are for the Monte Carlo simulation and the solid lines are for the analytical results. 


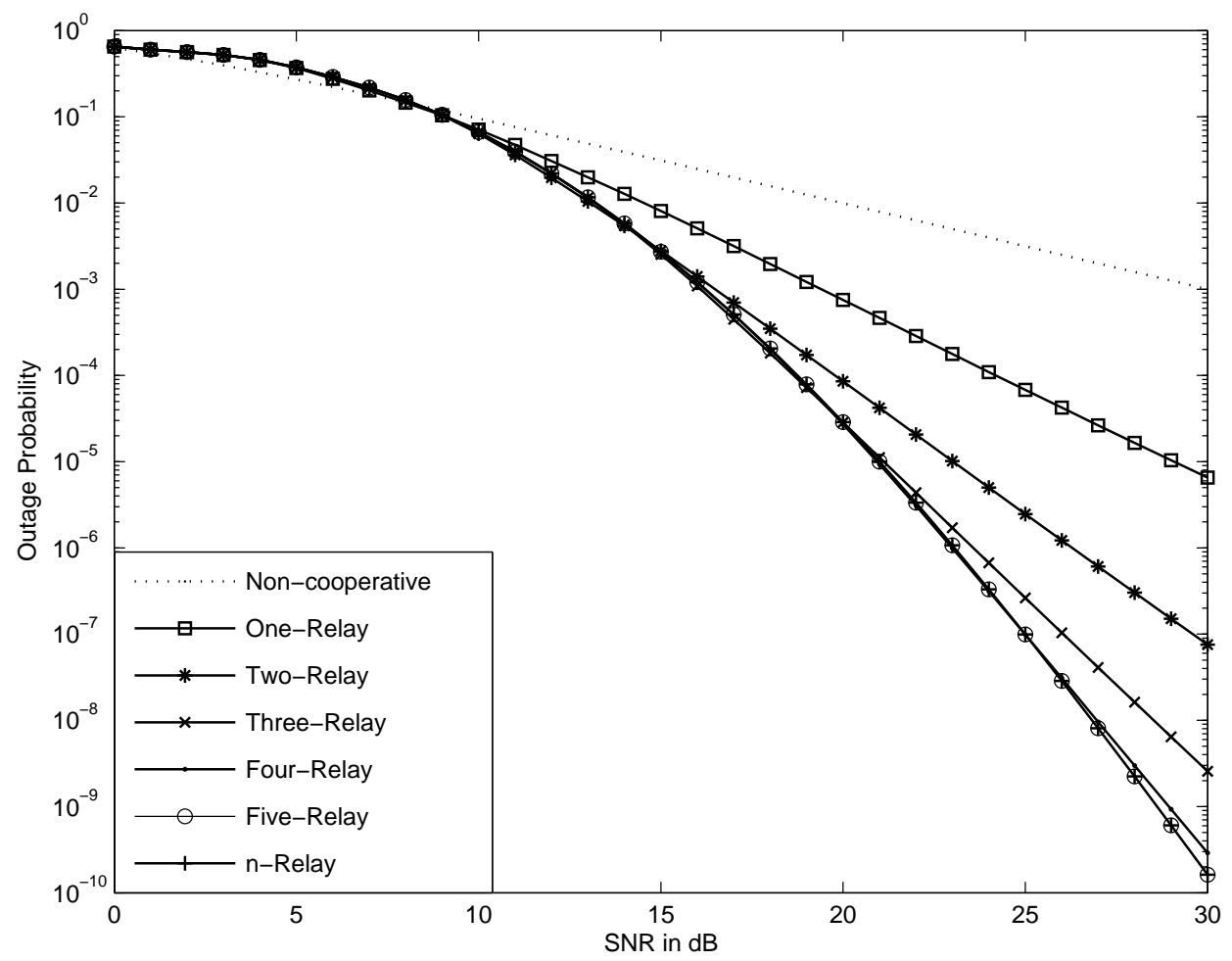

Fig. 5. The outage probability vs SNR. The dotted line is for the non cooperative scheme and the solid lines are for the relaying scheme with Type IV CSI.

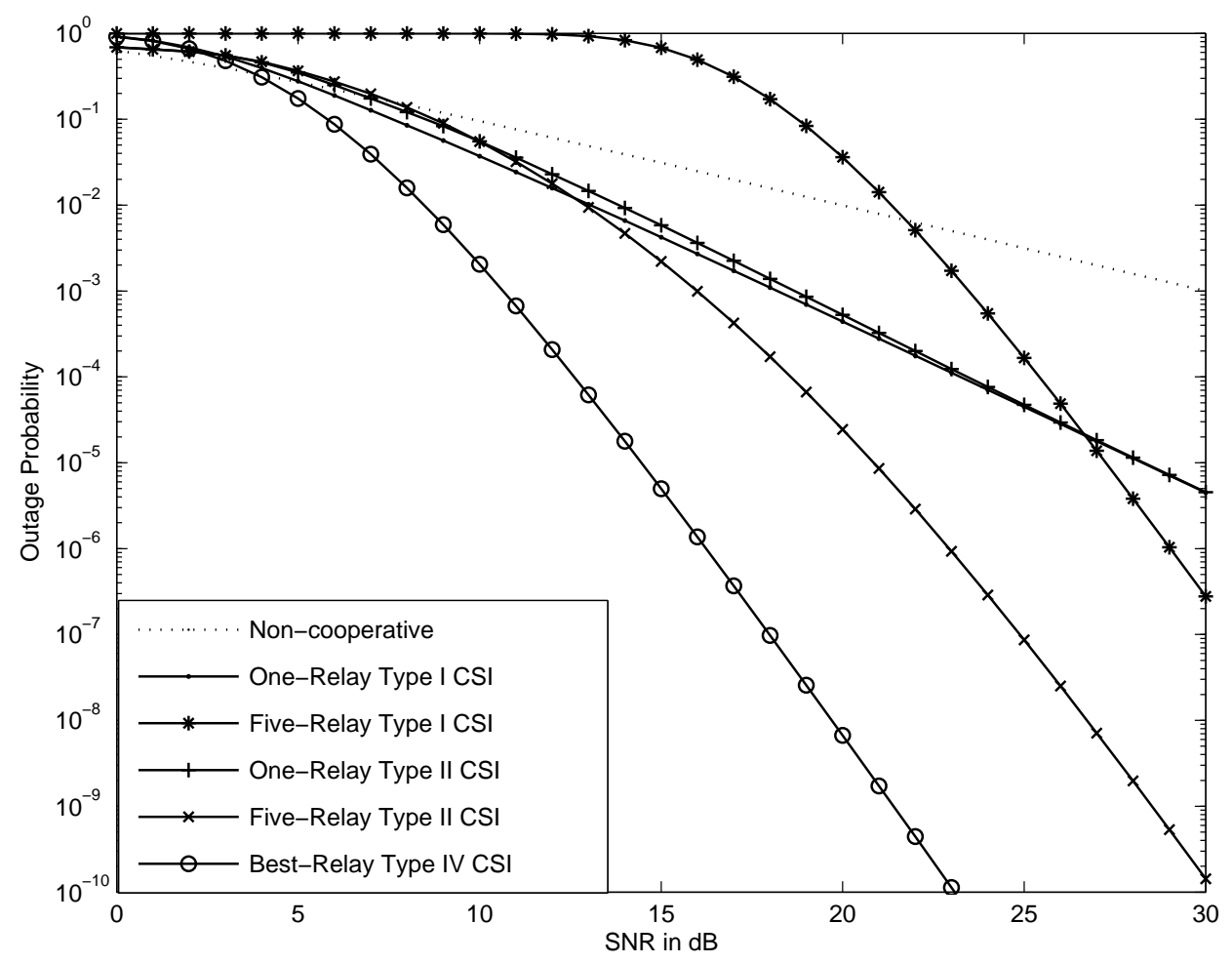

Fig. 6. The outage probability vs SNR. The dotted line is for the non cooperative scheme and the solid lines are for the proposed relaying schemes. 
where $\mathbf{y}=\left[\begin{array}{llll}y_{0} & y_{1} & \cdots & y_{k}\end{array}\right]^{T}, \mathbf{h}=\left[\begin{array}{llll}h_{0} & h_{1} & \cdots & h_{k}\end{array}\right]^{T}$ and $\mathbf{w}=\left[\begin{array}{llll}w_{0} & w_{1} & \cdots & w_{k}\end{array}\right]^{T}$. Hence the mutual information can be written as

$$
\mathcal{I}=\frac{1}{n+1} \log \left(1+\rho \sum_{i=0}^{k} y_{i}\right),
$$

where the factor $\frac{1}{n+1}$ is due to the fact that relaying transmission will consume $n$ extra channel uses compared with direct transmission. Furthermore, we obtain

$$
P(\mathcal{I}<R)=P\left(\sum_{i=0}^{k} y_{i}<\gamma_{n}\right)
$$

which needs the PDF of the variable $\sum_{i=0}^{k} y_{i}$. Since Raleigh fading is assumed, $\sum_{i=0}^{k} y_{i}$ will be Chi-square distributed with $2(k+1)$ degrees of freedom, whose PDF is $f_{\text {sum }}(z)=\frac{z^{k} e^{-z}}{k !}$. Hence we have

$$
\begin{aligned}
P(\mathcal{I}<R) & =1-e^{-\lambda \gamma_{n}} \sum_{i=0}^{k} \frac{\left(\lambda \gamma_{n}\right)^{i}}{i !} \\
& =1-e^{-\lambda \gamma_{n}}\left(e^{\lambda \gamma_{n}}-\sum_{i=k+1}^{\infty} \frac{\left(\lambda \gamma_{n}\right)^{i}}{i !}\right) \\
& =e^{-\lambda \gamma_{n}} \sum_{i=k+1}^{\infty} \frac{\left(\lambda \gamma_{n}\right)^{i}}{i !} \\
& \approx \frac{\left(\lambda \gamma_{n}\right)^{k+1}}{(k+1) !}
\end{aligned}
$$

where the second and third equations use the exponential expansion. The probability of those events $\mathcal{O}_{k}$ can be obtained by divided into three categories.

A. $P\left(\mathcal{O}_{k}\right)$ for $1 \leq k \leq(n-1)$

The probability for these events can be written as

$$
\begin{aligned}
P\left(\mathcal{O}_{k}\right) & =P\left(x_{(N-k)}<\gamma_{n}, x_{(N-k+1)}>\gamma_{n}\right) \\
& =\int_{0}^{\gamma_{n}} \int_{\gamma_{n}}^{\infty} f\left(x_{(N-k)}, x_{(N-k+1)}\right) d x_{(N-k)} d x_{(N-k+1)} .
\end{aligned}
$$

The evaluation requires the joint PDF of two ordered variables which can be expressed as

$$
f\left(x_{(N-k)}, x_{(N-k+1)}\right)=\frac{N !}{(N-k-1) !(k-1) !} f\left(x_{(N-k)}\right) f\left(x_{(N-k+1)}\right)\left[F\left(x_{(N-k)}\right)\right]^{N-k-1}\left[1-F\left(x_{(N-k+1)}\right)\right]^{k-1}(33
$$

So by using order statistics, the outage probability can be obtained as

$$
\begin{aligned}
P\left(\mathcal{O}_{k}\right) & =\int_{0}^{\gamma} \int_{\gamma}^{\infty} f\left(x_{N-k}, x_{N-k+1}\right) d x_{N-k} d x_{N-k+1} \\
& =\frac{N !}{(N-k-1) !(k-1) !} \int_{0}^{\gamma} \lambda e^{-\lambda x_{N-k}}\left[1-e^{-\lambda x_{N-k}}\right]^{N-k-1} d x_{N-k} \\
& \int_{\gamma}^{\infty} \lambda e^{-\lambda x_{N-k+1}}\left[e^{-\lambda x_{N-k+1}}\right]^{k-1} d x_{N-k+1} \\
= & \frac{N !}{(N-k) !(k) !}\left[1-e^{-\lambda \gamma}\right]^{N-k}\left[e^{-\lambda \gamma}\right]^{k}
\end{aligned}
$$


Again with the help of the exponential expansion, the outage probability can be approximated at high SNR as

$$
\begin{aligned}
P\left(\mathcal{O}_{k}\right) & \approx \frac{N !}{(N-k) !(k) !}[\lambda \gamma]^{N-k}[1-\lambda \gamma]^{k} \\
& \approx \frac{N !}{(N-k) !(k) !}[\lambda \gamma]^{N-k}
\end{aligned}
$$

B. $P\left(\mathcal{O}_{k}\right)$ for $k=0$

The evaluation of the probability $P\left(\mathcal{O}_{k}\right)$ requires the PDF of the largest variable $x_{(N)}$ which can be written as

$$
f_{x_{(N)}}(x)=N f(x)[F(x)]^{N-1}
$$

and we have

$$
\begin{aligned}
P\left(\mathcal{O}_{0}\right) & =P\left(x_{(N)}<\gamma_{n}\right)=\int_{0}^{\gamma_{n}} f_{x_{N}}(x) d x \\
& =\int_{0}^{\gamma_{n}} N f(x)[F(x)]^{N-1} d x \\
& =\left[1-e^{-\lambda \gamma_{n}}\right]^{N} .
\end{aligned}
$$

Note that $P\left(\mathcal{O}_{0}\right)$ can expressed as a special case of (34) for $k=0$.

C. $P\left(\mathcal{O}_{k}\right)$ for $k=n$

One way to find $P\left(\mathcal{O}_{n}\right)$ is to use the following expression

$$
P\left(\mathcal{O}_{n}\right)=P\left(x_{(N-n+1)}>\gamma_{n}\right)=\int_{0}^{\gamma_{n}} f_{x_{(N-n+1)}}(x) d x
$$

where $f_{x_{N-n+1}}(x)$ could be found by using order statistics. However its result is implicit and difficult to use. So we use an alternative way to find it by dividing $\mathcal{O}_{n}$ into the following $N-n+1$ independent events.

$$
\mathcal{O}_{n}:\left\{x_{(N-n+1)}>\gamma_{n}\right\}=\left\{\begin{array}{lll}
\mathcal{O}_{n, n} & x_{(N-n)}<\gamma_{n} & x_{(N-n+1)}>\gamma_{n} \\
\mathcal{O}_{n, n+1} & x_{(N-(n+1))}<\gamma_{n} & x_{(N-n)}>\gamma_{n} \\
\vdots & \vdots & \vdots \\
\mathcal{O}_{n, n+m-1} & x_{(N-(n+m-1))}<\gamma_{n} & x_{(N-(n-2+m))}>\gamma_{n} \\
\vdots & \vdots & \vdots \\
\mathcal{O}_{n, N-1} & x_{(1)}<\gamma_{n} & x_{(2)}>\gamma_{n} \\
\mathcal{O}_{n, N} & & x_{(1)}>\gamma_{n}
\end{array}\right.
$$


It is interesting to find that $P\left(\mathcal{O}_{n, m}\right)$ can be calculated by using (34) for $n \leq m \leq(N-1)$, which means

$$
P\left(\mathcal{O}_{n, m}\right)=\frac{N !}{(N-m) !(m) !}\left[1-e^{-\lambda \gamma_{n}}\right]^{N-m}\left[e^{-\lambda \gamma_{n}}\right]^{m}, \text { for } \quad n \leq m \leq(N-1) .
$$

The only left event is $\mathcal{O}_{n, N-n+1}$ which can be expressed as

$$
P\left(\mathcal{O}_{n, N-n+1}\right)=P\left(x_{(1)}>\gamma_{n}\right)=\int_{\gamma_{n}}^{\infty} f_{x_{(1)}}(x) d x
$$

which needs the PDF of $x_{(1)}$ which can be expressed as

$$
f_{x_{(1)}}(x)=N f(x)[1-F(x)]^{N-1}
$$

and we have

$$
\begin{aligned}
P\left(\mathcal{O}_{n, N-n+1}\right) & =\int_{\gamma_{n}}^{\infty} N f(x)[1-F(x)]^{N-1} d x \\
& =-\left.[1-F(x)]^{N}\right|_{\infty} ^{\gamma_{n}}=\left[e^{-\lambda \gamma_{n}}\right]^{N} .
\end{aligned}
$$

Interestingly, it is also the special case of (41) with $m=N$.

Now the probability for $\mathcal{O}_{n}$ can be written as

$$
\begin{aligned}
P\left(\mathcal{O}_{n}\right) & =\sum_{m=n}^{N} P\left(\mathcal{O}_{n, m}\right)=\sum_{m=n}^{N} \frac{N !}{(N-m) !(m) !}\left[1-e^{-\lambda \gamma_{n}}\right]^{N-m}\left[e^{-\lambda \gamma_{n}}\right]^{m} \\
& \approx\left[\lambda \gamma_{n}\right]^{N-n} \sum_{m=n}^{N} \frac{N !}{(N-m) !(m) !}
\end{aligned}
$$

By combining (31), (34) and (45), the theorem is proved.

Proof for Theorem 3 : Recall that all the chosen $n$ relay nodes can decode the source information correctly. Hence the outage event will be either caused by the poor link quality between the relay and destination nodes, or there is no qualified relay node existing $n=0$. Hence the outage probability for this scheme can be written as

$$
P_{\text {out }}=\sum_{n=0}^{N} P\left(\mathcal{I}_{n}<\gamma_{n} \mid \mathbf{n}=n\right) P(\mathbf{n}=n)
$$

where the threshold is now a function of the number of the used relay node.

The first factor of the product in (46) is the same as the results developed before and can be written as

$$
\begin{aligned}
P\left(\mathcal{I}_{n}<\gamma_{n} \mid \mathbf{n}=n\right) & =1-e^{-\lambda \gamma_{n}} \sum_{i=0}^{n} \frac{\left[\lambda \gamma_{n}\right]^{i}}{i !} \\
& \approx \frac{\left[\lambda \gamma_{n}\right]^{n+1}}{(n+1) !}
\end{aligned}
$$


As illustrated by Fig. 7, the probability of the event that there are $n$ successful relaying nodes can be expressed as

$$
\begin{aligned}
P(\mathbf{n}=n) & =P\left[x_{N-n+1}>\gamma_{n}, x_{N-n}<\gamma_{n+1}\right] \\
& =\frac{N !}{(N-n) ! n !}\left[1-e^{-\lambda \gamma_{n+1}}\right]^{N-n}\left[e^{-\lambda \gamma_{n}}\right]^{n}, \quad 0<n<N
\end{aligned}
$$

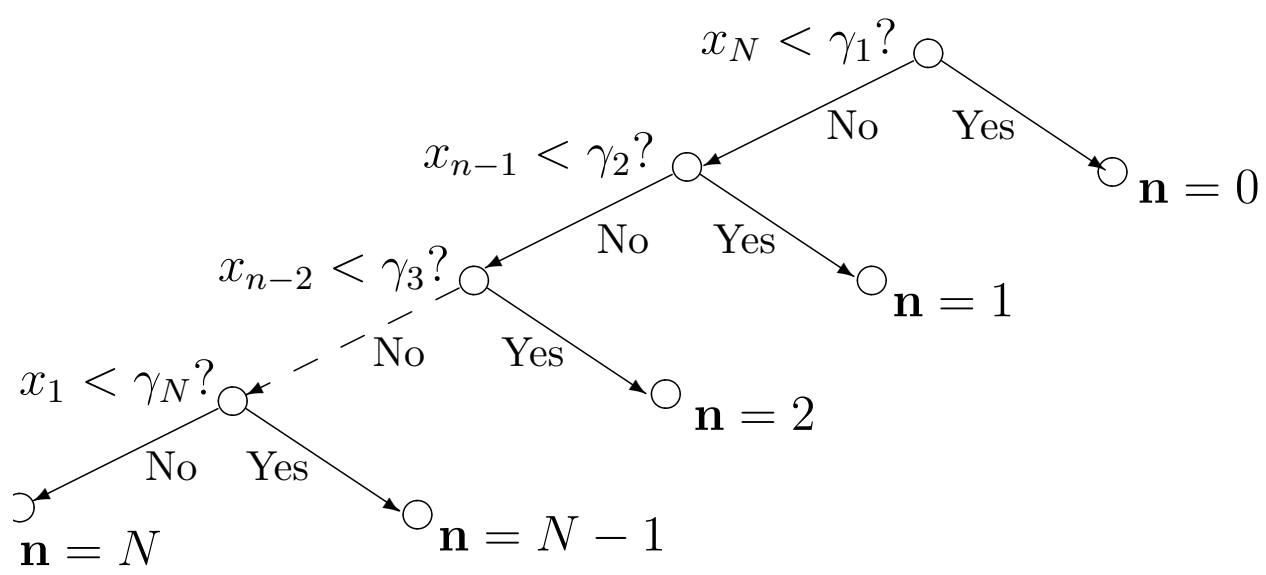

Fig. 7. The tree structure for the probability of the event that there are $n$ successful relaying nodes

The probability of the two specific events $\mathbf{n}=0$ and $\mathbf{n}=N$ is

$$
\begin{aligned}
P(\mathbf{n}=N) & =P\left[x_{1}>\gamma_{N}\right] \\
& =\int_{\gamma_{N}}^{\infty} N f(x)[1-F(x)]^{N-1} d x \\
& =\left.[1-F(x)]^{N}\right|_{\infty} ^{\gamma_{N}}=\left[e^{-\lambda \gamma_{N}}\right]^{N}
\end{aligned}
$$

and

$$
\begin{aligned}
P(\mathbf{n}=0) & =P\left[x_{N}<\gamma_{1}\right] \\
& =\int_{0}^{\gamma_{1}} N f(x) F(x)^{N-1} d x \\
& =\left.[F(x)]^{N}\right|_{0} ^{\gamma_{1}}=\left[1-e^{-\lambda \gamma_{1}}\right]^{N}
\end{aligned}
$$

By using the exponential expansion, the theorem is proved.

Proof for Theorem 11 : Recall that for the best relay scheme, the source node will choose the best relay among those nodes which can decode the source information correctly. Hence the outage probability can be written as

$$
P_{\text {out }}=\sum_{n=0}^{N} P\left(y_{0}+z_{(n)}<\gamma(1) \mid \mathbf{n}=n\right) P(\mathbf{n}=n)
$$

where $y_{0}$ is the channel fading from the source to the destination. 
Note that $z_{(n)}$ is the best one chosen from the $n$ relay candidates. So $z_{(n)}$ and $y_{0}$ are independent and hence the first factor of the product in (51) can be written as

$$
\begin{aligned}
P\left(y_{0}+z_{(n)}<\gamma(1) \mid \mathbf{n}=n\right) & =\int_{0}^{\gamma_{1}} \int_{0}^{\gamma_{1}-y} n f(z) F(z)^{n-1} d z f(y) d y \\
& =\int_{0}^{\gamma_{1}}\left[1-e^{-\lambda\left(\gamma_{1}-y\right)}\right]^{n} \lambda e^{-\lambda y} d y \\
& =\lambda e^{-\lambda \gamma_{1}} \int_{0}^{\gamma_{1}} \sum_{i=0}^{n} C_{n}^{i}(-1)^{i} e^{-(i-1) \lambda \psi} d \psi \\
& =\lambda e^{-\lambda \gamma_{1}}\left\{\sum_{\substack{i=0 \\
i \neq 1}}^{n} C_{n}^{i}(-1)^{i} \frac{1}{(i-1) \lambda}\left[1-e^{-(i-1) \lambda \gamma_{1}}\right]-n \lambda \gamma_{1}\right\}
\end{aligned}
$$

The probability of the event that there are $n$ successful relaying nodes can be expressed as

$$
\begin{aligned}
P(\mathbf{n}=n) & =P\left[x_{(N-n+1)}>\gamma(1), x_{(N-n)}<\gamma(1)\right] \\
& =\frac{N !}{(N-n) ! n !}\left[1-e^{-\lambda \gamma(1)}\right]^{N-n}\left[e^{-\lambda \gamma(1)}\right]^{n}, \quad 0<n<N
\end{aligned}
$$

The probability of the two events $\mathbf{n}=0$ and $\mathbf{n}=N$ is

$$
P(\mathbf{n}=N)=P\left[x_{(1)}>\gamma(1)\right]=\left[e^{-\lambda \gamma(1)}\right]^{N}
$$

and

$$
P(\mathbf{n}=0)=P\left[x_{(N)}<\gamma(1)\right]=\left[1-e^{-\lambda \gamma(1)}\right]^{N}
$$

which can be seen as two special cases of (54).

Hence the outage probability can be finally written as

$$
P_{\text {out }}=\sum_{n=0}^{N} \frac{N ! \lambda}{(N-n) ! n !}\left[1-e^{-\lambda \gamma(1)}\right]^{N-n}\left[e^{-\lambda \gamma(1)}\right]^{n+1}\left\{\sum_{\substack{i=0 \\ i \neq 1}}^{n} C_{n}^{i}(-1)^{i} \frac{1}{(i-1) \lambda}\left[1-e^{-(i-1) \lambda \gamma_{1}}\right]-n \lambda \gamma_{1}\right\}
$$

Although the exact expression of the outage probability is obtained, it is still not explicit how the performance is related with SNR. So it is desirable to find the high SNR approximation of the outage probability. Rewrite (53) as

$$
\begin{aligned}
P\left(y_{0}+z_{(n)}<\gamma(1) \mid \mathbf{n}=n\right) & =\int_{0}^{\gamma_{1}}\left[1-e^{-\lambda\left(\gamma_{1}-y\right)}\right]^{n} \lambda e^{-\lambda y} d y \\
& =\lambda e^{-\lambda \gamma_{1}} \int_{0}^{\gamma_{1}}\left[1-e^{-\lambda \psi}\right]^{n} e^{\lambda \psi} d \psi \\
& \approx \lambda \int_{0}^{\gamma_{1}}[\lambda \psi]^{n} d \psi \\
& =\frac{1}{n+1}\left(\lambda \gamma_{1}\right)^{n+1} .
\end{aligned}
$$


Substituting (58) into (51), we obtain

$$
\begin{aligned}
P_{\text {out }} & =\sum_{n=0}^{N} P\left(y_{0}+z_{(n)}<\gamma(1) \mid \mathbf{n}=n\right) P(\mathbf{n}=n) \\
& \approx \sum_{n=0}^{N} \frac{1}{n+1}\left(\lambda \gamma_{1}\right)^{n+1} \frac{N !}{(N-n) ! n !}\left[1-e^{-\lambda \gamma(1)}\right]^{N-n}\left[e^{-\lambda \gamma(1)}\right]^{n} \\
& \approx[\lambda \gamma(1)]^{N+1} \sum_{n=0}^{N} \frac{N !}{(N-n) !(n+1) !}
\end{aligned}
$$

\section{REFERENCES}

[1] T. S. Rappaport, Wireless communications: principles and practice. Prentice Hall, 1998.

[2] E. Telatar, "Capacity of multi-antenna gaussian channels," European Trans. Telecom., vol. 10, pp. 585-595, Nov./Dec. 1999.

[3] G. Foschini and M. Gans, "On limits of wireless communication in a fading environment when using multiple antennas," Wireless Personal Communications, vol. 6, no. 3, pp. 311-335, Mar. 1998.

[4] J. N. Laneman, D. N. C. Tse, and G. W. Wornell, "Cooperative diversity in wireless networks: Efficient protocols and outage behavior," IEEE Trans. Information Theory, vol. 50, pp. 3062-3080, Dec. 2004.

[5] A. Sendonaris, E. Erkip, and B. Aazhang, "User cooperation diversity-part I: system description,” IEEE Trans. communications, vol. 51, pp. 1927-1938, Nov. 2003.

[6] J. N. Laneman and G. W. Wornell, "Distributed space-time-coded protocols for exploiting cooperative diversity in wireless networks," IEEE Trans. Information Theory, vol. 49, pp. 2415-2425, Oct. 2003.

[7] Y. Zhao, R. Adve, and T. J. Lim, "Outage probability at arbitrary SNR with cooperative diversity," IEEE Comm. Letter, vol. 9, pp. 700-702, Aug. 2005.

[8] R. U. Nabar, H. Bolcskei, and F. W. Kneubuhler, "Fading relay channels : performance limits and space-time signal design," IEEE Trans. J. Sel. Areas Commun., vol. 22, pp. 1099-1109, Aug. 2004.

[9] K. Azarian, H. E. Gamal, and P. Schniter, "On the achievable diversity-multiplexing tradeoff in half-duplex cooperative channels," IEEE Trans. Information Theory, vol. 51, pp. 4152-4172, Nov. 2005.

[10] B. Zhao and M. C. Valenti, "Practical relay networks: A generalization of hybrid-ARQ," IEEE Journal on Select. Areas in Comm., vol. 23, pp. 7-18, Mar. 2005.

[11] A. Bletsas, A. Khisti, D. P. Reed, and A. Lippman, "A simple cooperative diversity method based on network path selection," IEEE Journal on Select. Areas in Comm., vol. 24, pp. 659-672, Mar. 2006.

[12] L. Zheng and D. N. C. Tse, "Diversisty and multiplexing : a fundamental tradeoff in multiple antenna channels," IEEE Trans. Information Theory, vol. 49, pp. 1073-1096, May 2003.

[13] D. N. C. Tse, P. Viswanath, and L. Zheng, "Diversity-multiplexing tradeoff in multiple-access channels," IEEE Trans. Information Theory, vol. 50, pp. 1859-1874, Sept. 2004. 